\title{
IX.-Note on a Method of effecting the substitution of Chlorine for Hydrogen in Organic Compounds.
}

\section{By Hugo MüLLER.}

Is the investigation of Burmese naphtha, made a few years ago by Mr. Warren De La Rue and myself, we endeavoured to obtain some definite iodine-compounds of the hydrocarbons contained in it, by acting upon them with mono-and ter-chloride of iodine, hoping that this reagent would act in this case in a similar way to that observed a short time before by Brown, in the case of pyromeconic acid. These experiments did not however give the expected result, but merely the same chlorine-compounds that were already obtailied by using chlorine only. The result was the same when iodine was dissolved in the hydro-carbon and chlorine passed into the deep violet-coluured solution. The only difference observable between the action of chlorine in presence of iodine and that of chlorine only, was that, in the former case, the action took place much more quickly than in the latter.

Having some time ago commenced a series of experiments with the vicw of obtaining from benzole the corresponding alcohol and acid, I had occasion to investigate the action of chlorine on this hydrocarbon.

Chlorine is absorbed to some extent by benzole, but if light be excluded, no pcrceptible action takes place until the benzole is heated to the boiling point ; hydrochloric acid is then liberated and chlorine-derivatives are formed, but very slowly. In this reaction two series of compounds are formed, namely,

$$
\begin{array}{lll}
\mathrm{C}_{6} \mathrm{H}_{6} \mathrm{Cl}_{2}, & \mathrm{C}_{6} \mathrm{H}_{6} \mathrm{Cl}_{4}, & \mathrm{C}_{6} \mathrm{H}_{6} \mathrm{Cl}_{6}, \text { and } \\
\mathrm{C}_{6} \mathrm{H}_{5} \mathrm{Cl}, & \mathrm{C}_{6} \mathrm{H}_{4} \mathrm{Cl}_{2}, & \mathrm{C}_{6} \mathrm{H}_{3} \mathrm{Cl}_{3}
\end{array}
$$

The experience gained in the above-mentioned experiments with Burmese naphtha, induced me to try upon a large scale the action of chlorine upon benzole in the presence of iodine.

When chlorine is passed into a saturated solution of iodine in benzole, it is completely absorbed, even when the current is moderately rapid, and after the violet solution has become brownish red, owing to the formation of monochloride of iodine, hydrochloric 
acid is liberated and chlorine-derivatives are rypidly formed. In this way, I have obtained the compounds $\mathrm{C}_{6} \mathrm{H}_{5} \mathrm{Cl}, \mathrm{C}_{6} \mathrm{H}_{4} \mathrm{Cl}_{2}$, $\mathrm{C}_{6} \mathrm{H}_{3} \mathrm{Cl}_{3}$, and wheu an excess of chlorine was used, a beautiful crystalline compound, which was previously obtained in small quantities on treating the hydrocarbons of Burmese naphtha in the same manner. It deserves to be noticed, that whereas when chlorine acts alone upon benzole, the chief products of the reaction are formed by its direct union with the hydrocarbon, the action of chlorine in presence of iodine gives rise to substitution-products and the evolution of hydrochloric acid.

I reserve the detailed description of the products of this reaction, and the method of obtaining them in a pure state for a future communication; what I wish especially to draw attention to upon this occasion is the remarkable effect of iodine in facilitating the action of chlorine upon organic compounds. I have already ascertained that many substances which are acted upon with great difficulty by chlorine alone, and some of them only with the aid of direct sunlight, yicld chlorine-products with great ease when acted upon, in the manner described, by chlorine in the presence of iodine,

I have found for instance that benzoic acid dissolved, together with some iodine in chloroform, is acted upon by chlorine very readily, with evolution of hydrochloric acid.

Bisulphide of carbon in which iodine is dissolved is acted upon by chlorine very frecly at its boiling point, giving chloride of carbon, chloride of sulphur, and a highly volatile substance having a very irritating and pungent odour, which is perhaps the chlorosulphide of carbon which Kolbe believed he had obtained by exposing sulphide of carbon to the action of chlorine in direct sunlight. The process $I$ have described promises to give this substance in sufficient quantity to admit of ascertaiuing its true nature.

When chlorine is passed into a solution of iodine in glacial acetic acid in the cold, terchloride of iodine is formed, but the acid is not acted upon. If however the acid is kept boiling whilst the chlorine is being passed into it, hydrochloric acid is given off and chloracetic acid is formed, even in the dark. This affords a ready means of obtaining chloracetic acid, a substance whose preparation according to the usual method requires the aid of strong sunlight, and is commonly very tedions. If the action of chlorine be continued, a higher chlorinated acid is obtained, which 
does not solidify even at $0^{\circ} \mathrm{C}$., and which appears to be bi-chloracctic acil.*

It seems likely, that in this reaction, the iodine acts merely as a carrier and condenser of the chlorine; the effect is very similar to that of some perchlorides, like pentachloride of antinony, and in some cases pentachloride of phosphorus, which net upon many organic substances as if they were mixtures of terchlorides with free chlorine.

I may instance here the formation of Dutch liquid from olefiant gas and pentachloride of antimony, according to Wöhler, and the case of the transformation of bisulphide of carbon into chloride of carbon and chluride of sulphur by means of pentachloride of antimony mentioned by $\mathrm{H}$ of mann.

On comparing the action of pentachloride of antimony with that of chlorine, in presence of iodine, upon the substances above mentioned, I find that the former agent is rather more energetic and possesses a tendency to produce the higher chlorinated derivatives.

Benzole added in small quantities to pentachloride of antimony, is acted upon with great violence, and there is at once formed a chlorinated derivative, which crystallises in beautiful white prisms nearly insoluble in alcohol, and not acted upon by alcoholic potash.

Succinic acid, when brought in contact with pentachloride of antimony, soon evolves hydrochloric acid, and if the reaction is suppurted by application of heat, chlorosuccinic acid is formed. If however the mixture of succinic acid with pentachloride of antimony be kept warm whilst a current of chlorine is passing into it, th: succinic acid is gradually decomposed into volatile products.

I am still engaged with the examination of some new products of these reactions, a full account of which I hope shortly to lay before the Society.

- Since the above was written I have found that, together with the chloracetic acid, there is invariably some iodacetic acid formed, and it appears that the formation of the latter is favoured by an excess of iodine used in the process. I have not ascertained whether the remarkable compounds lately obtained by Sehützenberger by the aetion of chloride of iodine upon acetate and benzoate of sodium are formed when chlorine acts upon acetic and benzoic acid in the presence of iodine; but as thèse compounds are already decomposed at $100^{\circ} \mathrm{C}$. their formation can hardly be expected under the circumstances. 